\title{
Are Tubules Generated by the 3a Protein Necessary for Cucumber Mosaic Virus Movement?
}

\author{
Tomas Canto and Peter Palukaitis \\ Virology Department, Scottish Crop Research Institute, Invergowrie, Dundee DD2 5DA, Scotland, U.K. \\ Accepted 13 August 1999.
}

\begin{abstract}
The 3a movement protein of cucumber mosaic virus (CMV), fused to the jellyfish green fluorescent protein (3aGFP) generated surface punctate aggregates as well as tubules protruding from infected tobacco and Nicotiana benthamiana protoplasts. Fluorescent tubules also appeared on the surface of protoplasts prepared from transgenic tobacco plants expressing 3a-GFP, indicating that the 3a protein is the only viral component required for the formation of the tubules. CMV with a mutation in the gene encoding the 3 a protein, M8 CMV, could infect tobacco systemically, but tubules were not detected protruding from infected protoplasts when the mutated 3a protein was fused to the GFP [(M8)3a-GFP]. This indicates that the ability of the 3a protein to generate tubules in the surface of protoplasts is not a function required for the spread of CMV in tobacco. On the other hand, the (M8)3a-GFP did not traffic through plasmodesmata interconnecting tobacco epidermal cells, in contrast to the wildtype 3a-GFP. This suggests that there may be a correlation between the ability of the 3 a protein to assemble tubules in protoplasts and its ability to promote movement within certain tissues.
\end{abstract}

Additional keywords: tubular structures, viral movement.

Movement of most plant viruses within the plant occurs in two forms: a slow cell-to-cell spread through the intercytoplasmic connections known as plasmodesmata, and a fast, long-distance spread through the vasculature (reviewed by Carrington et al. 1996), following the source-sink route of the metabolites in the phloem (Roberts et al. 1997). The viral factors involved in each type of movement and the manner in which the virus moves are not the same for different viruses. Cucumber mosaic virus (CMV) has two viral proteins that are required for cell-to-cell movement and also for long-distance movement: the 3 a movement protein (MP), and the viral coat protein (CP) (Suzuki et al. 1991; Boccard and Baulcombe 1993; Canto et al. 1997; Ryabov et al. 1999). Of the two, the role of the 3a protein in movement is better understood. It is known that the 3 a protein localizes to plasmodesmata in several infected Nicotiana spp. (Oparka et al. 1996; Vaquero et al. 1996; Blackman et al. 1998), where the MP modifies their

Corresponding author: Peter Palukaitis; Mailing address: Department of Virology, Scottish Crop Research Institute, Invergowrie, Dundee DD2 5DA, Scotland, U.K.; Telephone: +44 1382568 523; Fax: +44 1382562 426; E-mail: ppaluk@scri.sari.ac.uk gating capacity in both mesophyll and epidermal cells (Vaquero et al. 1994; Ding et al. 1995; Canto et al. 1997). The 3a protein can also traffic itself through these plasmodesmata (Ding et al. 1995; Canto et al. 1997; Itaya et al. 1997). The viral RNA is somehow transported through plasmodesmata by the 3 a protein, presumably by making use of the nucleic acidbinding and NTP-binding properties of the MP ( $\mathrm{Li}$ and Palukaitis 1996).

Many other viruses require both the MP and the viral $\mathrm{CP}$ for cell-to-cell movement, including members of the Caulimoviridae, Comoviridae, Nepoviridae, and Bunyaviridae (Linstead et al. 1988; van Lent et al. 1990; Weiczorek and Sanfaçon 1993; Kormelink et al. 1994). In the case of cowpea mosaic virus (CPMV), tomato spotted wilt virus (TSWV), or cauliflower mosaic virus (CaMV), extensive studies have shown the occurrence of tubules protruding from infected protoplasts. These tubular structures reacted immunologically with antisera against the MP of the corresponding virus (van Lent et al. 1991; Perbal et al. 1993; Thomas and Maule 1995; Kasteel et al. 1997b; Kikkert et al. 1997). In addition, electron microscopy (EM) showed tubular structures traversing the cell wall, connecting the cytoplasms of adjacent cells, in sections of infected plant tissues (Kasteel et al. 1997b; Perbal et al. 1993; Storms et al. 1995). Particulate structures were also present within these tubules, similar in size to virus particles for both CPMV and CaMV, both in plant tissue and in protoplasts (van Lent et al. 1990, 1991; Perbal et al. 1993). Such particles were not observed for TSWV (Storms et al. 1995). These data suggest that for some of these viruses cell-to-cell movement could occur via the tubular structures generated by the MP. The assembly of tubules in infected protoplasts by the MP seems to be related to this process.

To our knowledge, no tubular structures have been described in EM sections from tissues infected with viruses that only require a viral MP for local movement. It has been proposed that viruses that do not require the $\mathrm{CP}$ for local movement, like tobacco mosaic virus (TMV), would move through altered plasmodesmata in the form of ribonucleoprotein complexes between the viral RNA and the MP (Citovsky et al. 1990; Citovsky and Zambryski 1991).

Within the Bromoviridae, cell-to-cell viral movement is more complex. On the one hand, not all these viruses require the $\mathrm{CP}$ to move cell-to-cell; like CMV, alfalfa mosaic virus (AlMV) and brome mosaic virus (BMV) both require the MP and the viral CP to move cell to cell (van der Vossen et al. 1994; Schmitz and Rao 1996). However, the viral CP was found to be dispensable for cell-to-cell movement in the case 
of cowpea chlorotic mottle virus (CCMV) (Rao 1997). On the other hand, while no tubular structures have been observed spanning the cell wall in EM sections of tissues infected with CMV, tubular structures were found in tissues from Nicotiana benthamiana infected with olive latent virus 2 (OLV-2) (Grieco et al. 1999), which is also a member of the Bromoviridae. In the case of $\mathrm{CMV}$, the $3 \mathrm{a}$ protein that accumulated in the plasmodesmata did not form recognizable structures, and virus particles were not present (Ding et al. 1995; Vaquero et al. 1996; Blackman et al. 1998). In addition, CMV did not require the formation of virions to move cell to cell (Kaplan et al. 1998). In protoplasts, there is immunological evidence for tubules formed by the MPs of AlMV and BMV, and in both cases these contained viruslike particles (Kasteel et al. 1997a). This demonstrates that these structures are tubules rather than filaments. In the case of AlMV and OLV-2, the tagging of the MP with green fluorescent protein (GFP) showed that tubule assembly in protoplasts only required the MP (Zheng et al. 1997; Grieco et al. 1999).

The MPs of CMV (Li and Palukaitis 1996), BMV (Jansen et al. 1998), and AlMV (Schoumacher et al. 1992a, 1992b) all bind single-stranded RNA in vitro, similar to the MP of TMV (Citovsky et al. 1990), which does not require a CP for cell-tocell movement, or move cell-to-cell via tubules. Thus, it is not clear if there are alternative movement pathways or processes available to viruses within the Bromoviridae, whether they all share the same mechanisms, or whether different viruses have evolved to utilize one or another mechanism, but still retain certain atavistic functions due to the pleiotropic nature of the MPs. Here, we determine whether CMV, like BMV, AlMV, or OLV-2, retains the ability to generate tubulelike projections from the surface of protoplasts, whether the MP is the only protein essential for the generation of such tubules, and whether the ability to generate tubules is a requirement for CMV movement.

\section{RESULTS}

CMV induces protruding tubular structures

in $N$. tabacam (tobacco) and $N$. benthamiana protoplasts.

To determine whether CMV also induces the formation of tubules on the surface of protoplasts from host plants, as do other viruses in the Bromoviridae (Kasteel et al. 1997a; Zheng et al. 1997), tobacco and $N$. benthamiana protoplasts were electroporated with CMV transcript RNAs 1 and 2, together with transcript RNA 3 expressing the 3 a protein as a fusion to the GFP (Canto et al. 1997; Fig. 1A). At $24 \mathrm{~h}$ post inoculation (hpi), GFP-derived fluorescence was found in infected protoplasts, mostly associated with the cell membrane, forming discrete, dense aggregates of fluorescent material (Fig. 2B). The pattern of distribution of these aggregates varied among individual protoplasts. At $24 \mathrm{hpi}$, some protoplasts showed tubules protruding from the surface. These were short and originated from the dense fluorescent aggregates on the surface (Fig. 2B, arrows). At 48 hpi, tubules were more apparent in many protoplasts and, in general, longer, although length, like number of tubules and their distribution within individual cells, showed great variability (Fig. 2A; Table 1). In some cases, the punctate aggregates and tubules distributed uniformly on the surface of the cell, as shown by serial sections taken through infected protoplasts (e.g., Fig. 2A, sections 1-
4). In other cases, they accumulated in defined areas (Fig. 2A and B). Variability in the number of protoplasts displaying tubules versus the total of infected (fluorescent) protoplasts was also observed from experiment to experiment (Table 1). This may be due to the lability of these tubular structures. They were often found detached from the surface of the protoplast, moving freely in the medium. Any sudden motion of the protoplast culture could reduce the number of tubules attached to the protoplasts as well as their length (data not presented).

\section{The 3a protein is the only viral factor required} for the generation of tubules in protoplasts.

To determine whether the viral $\mathrm{CP}$, or any other viral component, was also involved in the formation of the tubules, two

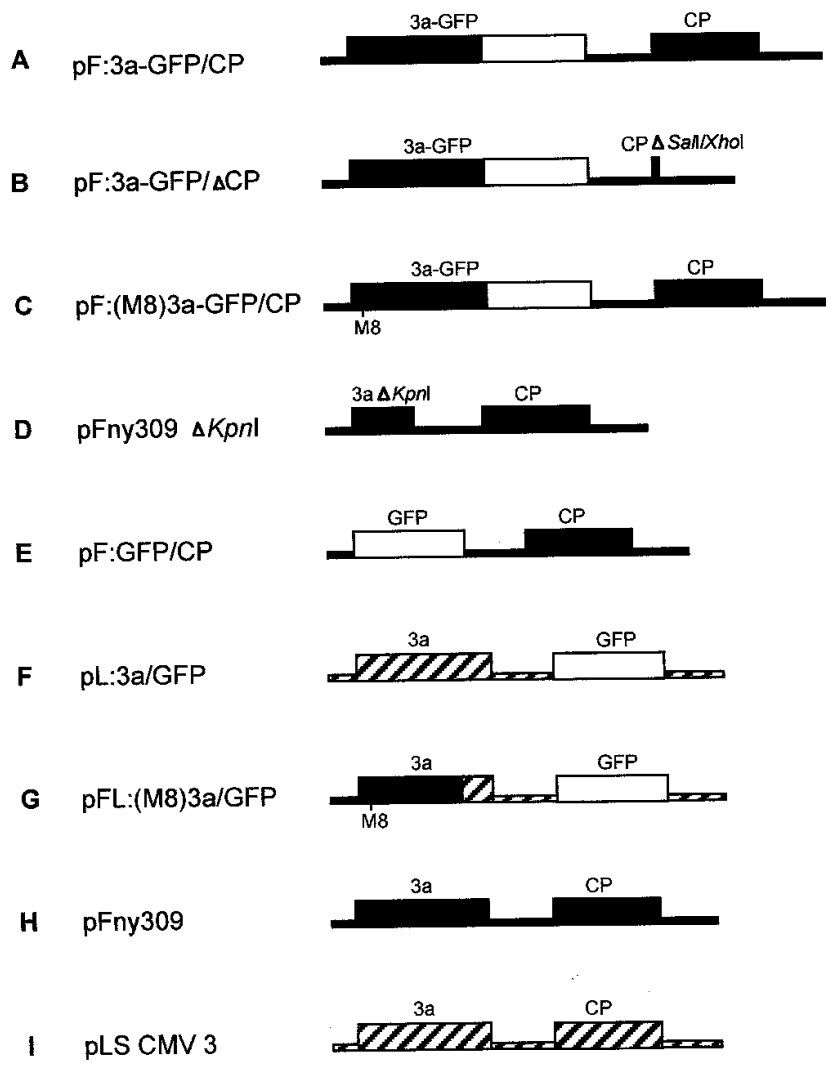

Fig. 1. Schematic representation of cucumber mosaic virus (CMV) RNA 3 transcripts used in this study. Transcripts were inoculated together with Fny-CMV transcript RNAs $1+2$. RNA 3 transcripts were derived from the following constructs: (A) $\mathrm{pF}: 3 \mathrm{a}-\mathrm{GFP} / \mathrm{CP}$, generating a modified Fny-CMV (solid rectangles) transcript RNA 3 that expresses the 3 a protein as a fusion to the green fluorescent protein (GFP) (open rectangle); (B) $\mathrm{pF}$ : $3 \mathrm{a}-\mathrm{GFP} / \triangle \mathrm{CP}$, similar to construct $\mathrm{pF}$ :3a-GFP/CP, but in which most of the coat protein (CP) gene has been deleted; (C) $\mathrm{pF}:(\mathrm{M} 8) 3 \mathrm{a}-$ $\mathrm{GFP} / \mathrm{CP}$, similar to construct $\mathrm{pF}: 3 \mathrm{a}-\mathrm{GFP} / \mathrm{CP}$, but expressing the mutated M8 CMV 3a protein fused to the GFP (open rectangle); (D) pFny309 $\Delta K p n \mathrm{I}$, in which two-thirds (501 nucleotides) of the 3a protein gene has been deleted; (E) pF:GFP/CP, generating a modified Fny-CMV RNA 3 in which the $3 \mathrm{a}$ gene has been replaced by the gene encoding the GFP (open rectangle); (F) construct $\mathrm{pL}: 3 \mathrm{a} / \mathrm{GFP}$, generating a modified LSCMV RNA 3 (crosshatched rectangles) in which the gene encoding the $\mathrm{CP}$ has been replaced by the GFP gene (open rectangle); (G) pFL: (M8)3a/GFP, where the chimeric Fny/LS encoded 3a protein has mutations at amino acids 20 and 21, both Asp to Ala, in the Fny-CMV 3a protein (M8); and (H) clone pFny309, generating wild-type (WT) FnyCMV RNA 3; and (I) clone pLS309, generating WT LS-CMV RNA 3. 


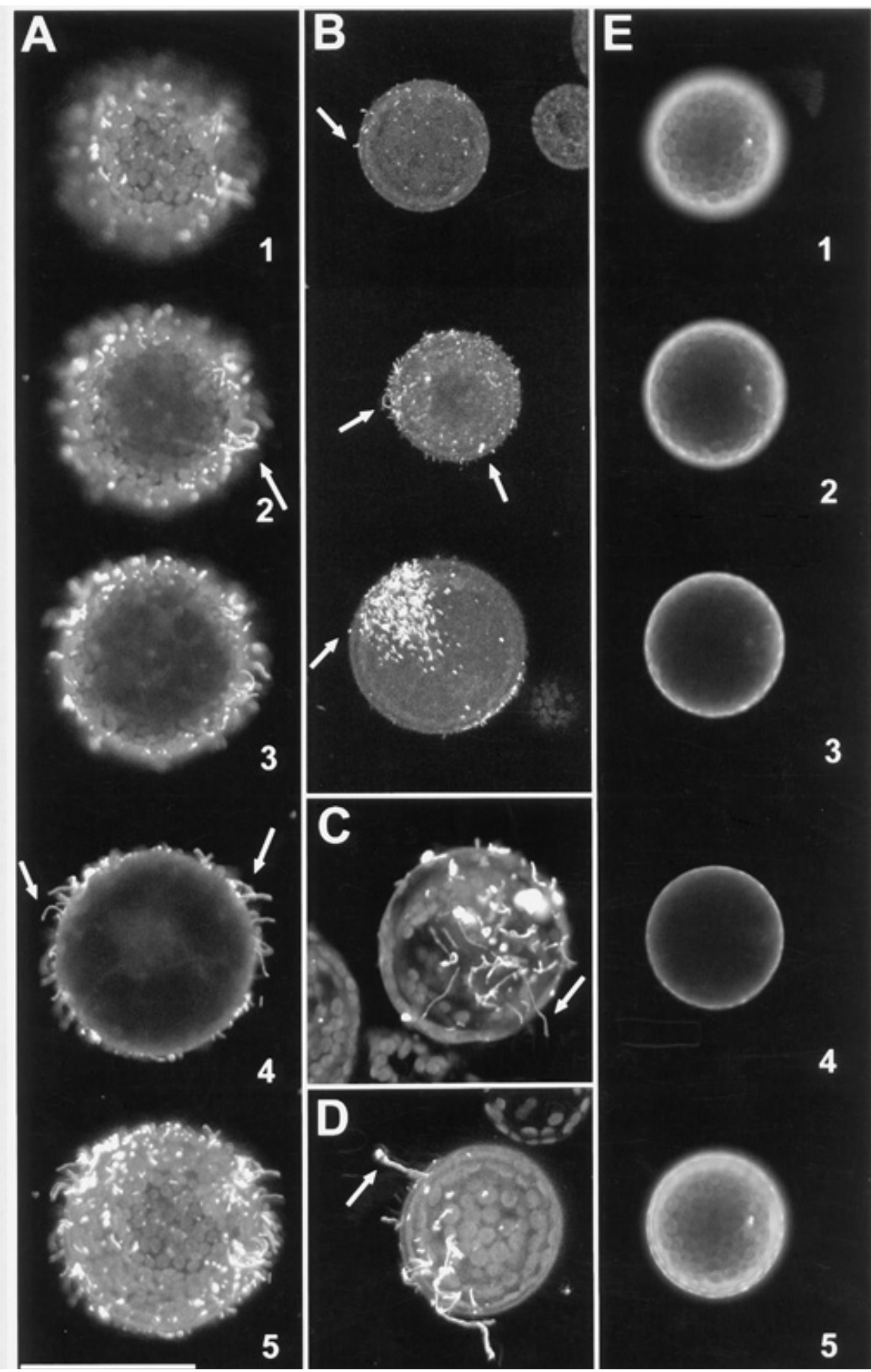

Fig. 2. Images obtained by confocal electron microscopy of tobacco protoplasts in which the 3a protein is expressed as a fusion to the green fluorescent protein (GFP). A, B, C, and E, Protoplasts were electroporated with cucumber mosaic virus (CMV) transcript RNAs 1 and 2, plus (A and B) transcript RNA 3 derived from construct $\mathrm{pF}: 3 \mathrm{a}-\mathrm{GFP} / \mathrm{CP}$, expressing wild-type $3 \mathrm{a}-\mathrm{GFP}, \mathbf{( C )}$ transcript RNA 3 from construct $\mathrm{pF}: 3 \mathrm{a}-\mathrm{GFP} / \Delta \mathrm{CP}$, in which the viral CP is not produced, and (E) transcript RNA 3 derived from construct $\mathrm{pF}:(\mathrm{M} 8) 3 \mathrm{a}-\mathrm{GFP} / \mathrm{CP}$, which expresses a mutated 3a protein. D, A protoplast isolated from transgenic tobacco plants constitutively expressing 3a-GFP. B, Images were taken at $24 \mathrm{~h}$ post inoculation (hpi). All the other images were taken at $48 \mathrm{hpi}$. A and $\mathbf{E}$, Images 1 to 4 represent serial sections (at intervals of approx. $8 \mu \mathrm{m}$ ) of a single protoplast. Image 5 is a composition of the serial images. Arrows show some of the GFP-fluorescent tubules and protrusions. Bar represents $100 \mu \mathrm{m}$. 
approaches were taken. In the first approach, a CMV variant was engineered that contained the 3a-GFP fusion gene in RNA 3, as well as a deletion of most of the CP gene (construct $\mathrm{pF}: 3 \mathrm{a}-\mathrm{GFP} / \triangle \mathrm{CP}$; Fig. 1B). Protoplasts electroporated with transcript RNA 3 derived from this construct, together with transcript RNAs 1 and 2, also exhibited fluorescent tubules on their surface, indistinguishable by confocal microscopy from those induced by virus expressing intact $\mathrm{CP}$ (Fig. $2 \mathrm{C}$ versus $\mathrm{A}$ and $\mathrm{B}$ ). This indicates that the viral $\mathrm{CP}$, either free or in the form of virions, is not required for the generation of tubules. Furthermore, in a second approach, protoplasts were obtained from transgenic tobacco plants that expressed high levels of 3a-GFP. Tubules were synthesized de novo in a fashion similar to that observed in nontransformed protoplasts infected

Table 1. Proportion of mesophyll protoplasts exhibiting tubular structures after electroporation with cucumber mosaic virus (CMV) expressing 3a-GFP

\begin{tabular}{|c|c|c|c|c|}
\hline $\begin{array}{l}\text { Type of 3a-GFP } \\
\text { fusion }\end{array}$ & $\begin{array}{c}\text { Tub/Inf } \\
24 \text { hpi }\end{array}$ & $\begin{array}{c}\% \\
\text { inf }^{b}\end{array}$ & $\begin{array}{c}\text { Tub/Inf } \\
48 \text { hpi }\end{array}$ & Host \\
\hline \multicolumn{5}{|l|}{ WT 3a-GFP } \\
\hline Experiment 1 & $10 / 100$ & 18 & $27 / 112$ & Nicotiana benthamiana \\
\hline Experiment 2 & & 39 & $14 / 100$ & N. benthamiana \\
\hline Experiment 3 & $53 / 100$ & 50 & $35 / 100$ & Tobacco \\
\hline Experiment 4 & $51 / 102$ & 44 & $85 / 100$ & Tobacco \\
\hline \multicolumn{5}{|l|}{ (M8)3a-GFP } \\
\hline Experiment 1 & $0 / 224$ & 42 & $0 / 249$ & N. benthamiana \\
\hline Experiment 2 & & 66 & $0 / 100$ & N. benthamiana \\
\hline Experiment 3 & $0 / 100$ & $>50$ & $0 / 100$ & Tobacco \\
\hline Experiment 4 & $0 / 100$ & 30 & $0 / 100$ & Tobacco \\
\hline
\end{tabular}

${ }^{a}$ Number of protoplasts with tubules over the total of infected (fluorescent) protoplasts at 24 or 48 hours post inoculation (hpi).

b Percentage of protoplasts infected (fluorescent).

c Protoplasts infected by CMV expressing the wild-type 3 a protein fused to the green fluorescent protein (GFP).

${ }^{\mathrm{d}}$ Protoplasts infected by CMV expressing the mutant M8 3a protein fused to the GFP. with virus expressing 3a-GFP (Fig. 2D versus A, B, and C). Thus, the $3 \mathrm{a}$ protein is the only viral component required for the generation of tubules in protoplasts.

\section{Impaired ability of the 3a protein to assemble tubules in tobacco and $N$. benthamiana protoplasts did not affect the systemic spread of CMV in these species.}

The biological significance of the generation of tubules by the $3 \mathrm{a}$ protein in plant protoplasts, and specifically the relationship of the generation of tubules to the viral movement processes, was investigated with a number of CMV 3a protein mutants characterized with regard to movement in tobacco and $N$. benthamiana plants ( $\mathrm{Li}$ 1995). The genes encoding mutated 3a proteins were fused to the gene encoding the GFP, and RNA 3 derived from each of the constructs was assayed for the formation of tubules in protoplasts. One of these mutants, M8 CMV, that could move both cell to cell and systemically in whole plants, could not induce the formation of tubules in protoplasts when the mutated MP was fused to the GFP (Table 1). M8 CMV encodes two amino acidic substitutions at positions 20 and 21 of the $3 \mathrm{a}$ gene, both Asp to Ala. The (M8)3a-GFP fusion protein, expressed from construct $\mathrm{pF}$ :(M8)3a-GFP/CP (Fig. 1C), failed to induce the formation of fluorescent tubules in protoplasts (Fig. 2E). Tubules were not detected at any time during the observation period, from 12 hpi to more than 7 days post inoculation (Table 1 and data not shown).

The absence of tubules induced by the (M8)3a-GFP fusion protein was not due to a lower stability of the fusion protein in protoplasts. Western blot (immunoblot) analysis revealed that the 3a-GFP was present in similar amounts in protoplasts infected with virus expressing either the wild-type (WT) 3aGFP or the mutated (M8)3a-GFP (Fig. 3A, lane 1 versus lane 2). Interestingly, the antiserum also reacted with a protein band of about $30 \mathrm{kDa}$, running slightly faster than the WT $3 \mathrm{a}$ protein (see Figure 3, lane 2). This suggests that some degra-

\section{A B}

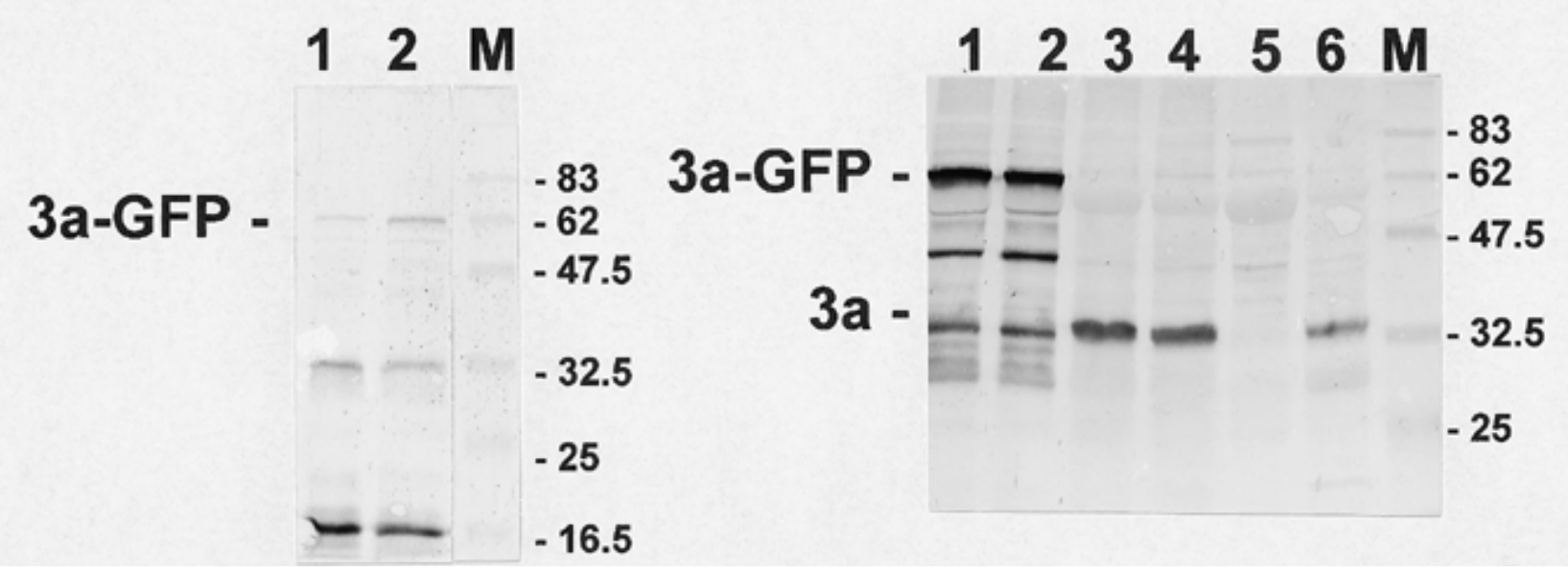

Fig. 3. Western blot (immunoblot) analysis of the accumulation and stability of 3a-GFP. A, Proteins were extracted from protoplasts electroporated with Fny-CMV transcript RNAs 1 and 2, and transcript RNA 3 derived from either (lane 1) construct pF:3a-GFP/CP or (lane 2) construct pF:(M8)3a-GFP/CP. B, Comparative levels of accumulation of $3 \mathrm{a}-\mathrm{GFP}$ and $3 \mathrm{a}$ protein in extracts from transgenic and infected plants. Levels of accumulation of (lanes 1 and 2) 3a-GFP and (lanes 3 and 4) 3a proteins are shown for two 3a-GFP- and two 3a-transgenic tobacco plants, respectively, as well as of 3a protein in nontransformed tobacco infected with Fny-CMV (lane 6). Lane 5 contains proteins extracted from noninoculated, nontransformed tobacco. Membranes were probed with a polyclonal antibody against the 3a protein (Gal-On et al. 1994). M, molecular weight markers. 
dation of both fusion proteins occurred, and opens the possibility that the tubules contained not only the 3a-GFP fusion but also this partial degradation product with 3 a protein epitopes.

When extracts from 3a-GFP transgenic plants were analyzed with Western blots, the results were similar. In addition to the band corresponding to the fusion product (Fig. 3B, lanes 1 and 2), the antiserum recognized a band that migrated slightly faster than that corresponding to the $3 \mathrm{a}$ protein, expressed either from the transgene in $3 \mathrm{a}$ transgenic plants or from virus in inoculated, nontransformed tobacco (Fig. 3B, lanes 1 and 2 versus 3, 4, and 6). The levels of accumulation of the transgenic 3a-GFP were higher than those exhibited by either the transgenic $3 \mathrm{a}$ protein alone, or the $3 \mathrm{a}$ protein in nontransformed tobacco infected with CMV (Fig. 3B, lanes 1 and 2 versus lanes 3,4 , and 6).

\section{The 3a-GFP fusion is not functional as an MP for CMV RNA.}

Transgenic plants expressing the 3 a protein were known to complement 3a-deficient CMV for movement (Kaplan et al. 1995). To determine whether the fusion with the GFP had any effect on the biological functions of the 3 a protein in movement, plants transgenic for the 3a-GFP were inoculated with 3a-deficient CMV ( $\triangle$ KpnI-CMV; Kaplan et al. 1995; Fig. 1D). Surprisingly, there was no complementation of CMV movement, either systemically (Fig. 4A, lanes 4 to 6 versus lane 2), or from cell to cell (data not shown). This result also suggests that the degradation product detected in the Western blot shown in Figure 3 cannot function in place of the intact $3 a$ protein in promoting viral movement.

To determine whether the lack of complementation for movement was due to interference by $3 a-G F P$ fragments in the transgenic plants expressing 3a-GFP, these plants were inoculated with movement-competent, WT virus. The WT virus moved in these plants as it did in nontransformed tobacco (Fig. 4B, lanes 1-4). This indicates that the transgene products were not blocking putative sites of binding for the virusencoded, WT 3a protein.

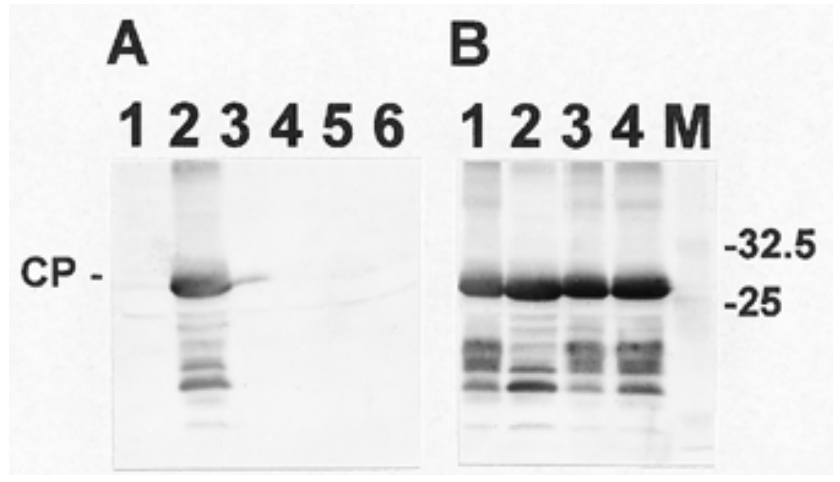

Fig. 4. Western blot (immunoblot) analysis of systemic accumulation of coat protein (CP) in plants infected by (A) a movement-deficient $\Delta K p n \mathrm{I}$ Fny-CMV, or (B) the movement-competent Fny-CMV. Proteins were extracted from (lane 1) nontransformed tobacco, (lane 2) 3a transgenic tobacco, and (lanes 3-4 in A, 3-6 in B) different 3a-GFP transgenic tobacco plants. The band (A, lane 3 ) at the $\mathrm{CP}$ position is spillover from lane 2. Membranes were probed with polyclonal antisera against the viral CP (Gal-On et al. 1994). M, molecular weight markers.
The impaired ability of 3a protein to assemble tubules in protoplasts correlated with an impaired ability of CMV to spread within specific tissues.

When CMV expressing WT 3a-GFP was inoculated to tobacco, infection remained confined to single epidermal cells (Canto et al. 1997). The same occurred with virus expressing (M8)3a-GFP (Fig. 5B). The WT 3a-GFP localized to plasmodesmata and moved to neighboring epidermal cells (Canto et al. 1997; Itaya et al. 1997; Fig. 5A, arrows). However, in the case of (M8)3a-GFP, fluorescence distributed more homogeneously within the cytoplasm, and possibly was also present inside the cellular vacuole, but did not spread to neighboring epidermal cells (Fig. 5B). This indicates that the (M8)3a-GFP was unable to traffic to neighboring epidermal cells as did the WT 3a-GFP.

To characterize the cell-to-cell movement of M8 CMV at the cytological level, an infection system was used containing two viral RNAs 3, each expressing GFP in place of either the 3a protein or the viral $\mathrm{CP}$, respectively (Fig. 1E and F). These two RNAs 3 were known to complement each other in trans, allowing the virus to move cell to cell (Canto et al. 1997; Fig. 5C). Thus, viral RNAs 1 and 2 were inoculated with two transcript RNAs 3 derived from constructs $\mathrm{pF}$ :GFP/CP and pFL:(M8)3a/GFP, the latter expressing a chimeric Fny/LSCMV 3a protein containing the M8 mutation (Fig. $1 \mathrm{E}$ and $\mathrm{G}$, respectively). The chimeric nature of the resulting 3 a protein was known not to alter the functionality of this protein (Zhang et al. 1994). The resulting virus could also spread in tobacco, creating clusters of fluorescent cells (Fig. 5D). However, the clusters contained mostly mesophyll cells (arrowheads in Figure 5D), with only one or two epidermal cells (arrow in Figure $5 \mathrm{D}$ and data not shown). This is in contrast to the results obtained when WT 3a protein was used instead of the M8 3a protein; i.e., the latter clusters contained large numbers of both epidermal and mesophyll cells (Fig. 5C versus D). The same results were observed in inoculated $N$. benthamiana plants (data not shown). Thus, the use of fluorescence microscopy and GFP demonstrated that, in tobacco and $N$. benthamiana, the (M8)3a-GFP fusion protein was unable to traffic through plasmodesmata interconnecting epidermal cells. This suggests that the virus containing the M8 mutation (M8 CMV) could not move through these same plasmodesmata either, but this virus could spread via plasmodesmata to and between mesophyll tissue.

\section{DISCUSSION}

GFP has been widely used as an in vivo marker for the detection of viruses in plant tissues and for the intracellular localization of GFP-tagged viral proteins. In the case of CMV, the neutral effect of the tagging with GFP on the localization and other properties of the $3 \mathrm{a}$ protein have been well documented (Oparka et al. 1996; Canto et al. 1997; Itaya et al. 1997; Blackman et al. 1998). In parallel studies involving EM immunolocalization of the WT 3a protein and confocal microscopy localization of 3a-GFP, completely consistent results were obtained (Blackman et al. 1998). The 3a protein fused to GFP was found to be capable of (i) localizing to plasmodesmata in epidermal cells, as did the WT 3a (Blackman et al. 1998), (ii) modifying the gating capacity of plasmodesmata in epidermal cells (Canto et al. 1997), (iii) trafficking through 
plasmodesmata to neighboring epidermal cells, even in the absence of viral RNA (Canto et al. 1997; Itaya et al. 1997), as did the WT 3a protein in mesophyll tissue (Ding et al. 1995), and (iv) generating tubules in protoplasts. Thus, it was surprising to find that the 3a-GFP fusion was unable to promote viral movement of a 3a-deficient CMV mutant. It is possible that the nucleic acid-binding ability of 3a-GFP or its specificity had been affected or abolished, or that some conformation required for trafficking RNA had been affected. A change in the conformation of the CMV MP to facilitate cell-to-cell trafficking has been proposed by Kragler et al. (1998) and Ryabov et al. (1999). Alternatively, the complex of 3a protein GFP and RNA may be beyond the size limitation of molecules that can be trafficked by the CMV MP.

The CMV MP fused to GFP formed punctate aggregates at the periphery of tobacco and $N$. benthamiana protoplasts. Subsequently, tubules of varying lengths emerged from the surface of such protoplasts from the sites of the punctate aggregates. This is similar to the situation observed during TMV infection in plants or in protoplasts of these two Nicotiana spp., where punctate structures of MP-GFP appeared at the cell periphery, although these were also associated with cortical endoplasmic reticula (Heinlein et al. 1998). However, in the case of
TMV only hairlike projections were present on the surface of protoplasts, while CMV, like other viruses in the Bromoviridae, generated long tubules (Kasteel et al. 1997a; Zheng et al. 1997; Grieco et al. 1999), similar to those generated by CaMV (Perbal et al. 1993) or CPMV (van Lent et al. 1991).

Using CP deletion mutants and transgenic plants expressing the $3 \mathrm{a}$ protein, we showed that the MP is the only viral factor required and sufficient for the induction of these tubular structures in protoplasts. This is similar to what has been described for CPMV, TSWV, AlMV, and OLV-2 (Wellink et al. 1993; Storms et al. 1995; Zheng et al. 1997; Grieco et al. 1999). Although the tubules induced by AlMV and CPMV were generated by the viral MPs in the absence of other viral components (Wellink et al. 1993; Zheng et al. 1997), when tubules were induced by virus replicating in the protoplast, viruslike particles were found inside the tubules (Kasteel et al. 1997a, 1997b). This indicated that these structures were actually tubules and not filaments. If the architecture of these structures remains the same whether virions are present or not, then the structures described for MP fused to GFP would also be tubules (Zheng et al. 1997; Grieco et al. 1999; this report).

In the case of CMV, the ability to generate tubules in protoplasts did not correlate with the presence of tubular structures
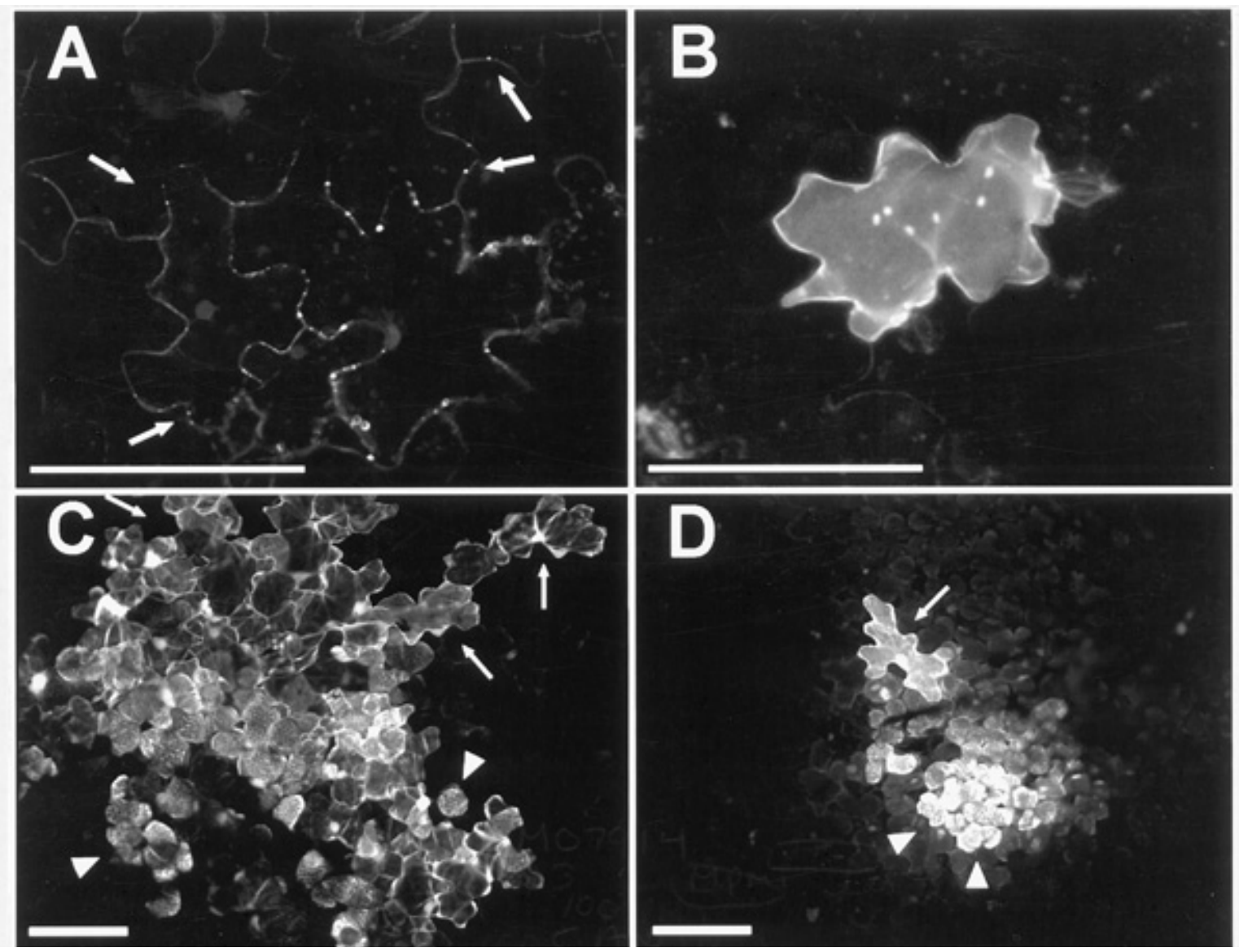

Fig. 5. Images obtained by confocal electron microscopy of inoculated tobacco leaves. A and $\mathbf{B}$, Infected epidermal cells from tobacco leaves that had been inoculated with Fny-CMV transcript RNAs 1 and 2, plus transcript RNA 3 derived from construct (A) pF:3a-GFP/CP and (B) pF:(M8)3a-GFP/CP. A, Fluorescence is localized in discrete spots within the cell wall and is also found in some of the neighboring epidermal cells (arrows). B, Fluorescence is distributed more homogeneously throughout the whole cell and it is not present in neighboring cells. $\mathbf{C}$ and $\mathbf{D}$, Images of fluorescent clusters of epidermal (arrows) and mesophyll (arrowheads) cells from tobacco leaves inoculated with transcript RNAs 1 and 2, plus a mixture of two transcript RNAs 3 derived from constructs (A) pF:GFP/CP and pL:3a/GFP or (B) pF:GFP/CP and pFL:(M8)3a/GFP. Only one infected epidermal cell is found in the cluster shown in $\mathbf{B}$, in contrast to several epidermal cells found in the cluster shown in $\mathbf{A}$. Bar represents $100 \mu \mathrm{m}$. 
traversing the cell walls of infected tissue (Ding et al. 1995; Blackman et al. 1998), unlike the situation with OLV-2 (Grieco et al. 1999). However, we have shown that the inability of the MP to induce tubules did not prevent CMV from moving systemically in two Nicotiana spp. The ability of the MP of M8 CMV to generate tubules was abolished, but the virus could still spread systemically in both tobacco and $N$. benthamiana plants. On the other hand, in contrast to the WT MP, the mutant (M8)3a-GFP fusion protein could not traffic through the plasmodesmata that interconnect epidermal tobacco cells. The lack of tubules associated with (M8)3a-GFP could be due to (i) an inability of the M8 MP to assemble (with host components) into the large punctate aggregates on the surface of the protoplasts (compare Figure 2E with B), (ii) an inability of the M8 MP to assemble into tubules, or (iii) extreme instability or lability of the tubules generated by the M8 MP.

In the case of M8 CMV, no tubules were generated in protoplasts, yet the virus still moved systemically in whole plants, with no delay in the appearance of symptoms, relative to the WT virus. Thus, the ability of the WT MP to induce tubules in protoplasts may be related to its ability to promote viral movement through plasmodesmata interconnecting tobacco epidermal cells, but not through plasmodesmata either connecting epidermal cells with mesophyll cells or between mesophyll cells. While it might even be possible that 3 a protein-induced tubules are actually generated in infected epidermal tissue, no such tubules were detected in EM sections of epidermal tissue (Blackman et al. 1998). Such tubules may only exist transiently in some tissues, or different plasmodesmata in different tissues may require different functions of the MP to promote viral movement through them. Alternatively, while it seems clear that the 3 a protein does not require the ability to form tubules to promote the cell-to-cell and systemic spread of CMV in two Nicotiana spp., the ability to generate tubules could represent an evolutionary remnant that allows the $3 \mathrm{a}$ protein to promote virus movement in certain tissues and/or hosts.

\section{MATERIALS AND METHODS}

Plants, viruses, plasmid constructs, and plant inoculation.

Tobacco (Nicotiana tabacum cv. Samsun NN) and Nicotiana benthamiana plants were maintained in the greenhouse. Transgenic tobacco expressing 3a-GFP, described by Itaya et al. (1997), were obtained from B. Ding (Oklahoma State University). The various plants were inoculated with either nonrecombinant virus (Fny-CMV) or RNA transcripts generated from the full-length cDNA clones pFny109, pFny209, corresponding to Fny-CMV RNAs 1 and 2 (Rizzo and Palukaitis 1990), plus RNA 3 transcripts generated from the following clones: pFny309, pF:GFP/CP, pL:3a/GFP, pF:3a-GFP/CP, pF:(M8)3a-GFP/CP, pFL:(M8)3a/GFP, pF:3a-GFP/ $\triangle \mathrm{CP}$, or pFny309 $\Delta K p n I$. Construct pFny309 corresponds to the WT Fny-CMV RNA 3 (Rizzo and Palukaitis 1990). Constructs $\mathrm{pF}: \mathrm{GFP} / \mathrm{CP}$ and $\mathrm{pL}: 3 \mathrm{a} / \mathrm{GFP}$ contain sequences of Fny- or LSRNA 3, respectively, modified so that the GFP gene is substituted for either the viral $3 \mathrm{a}$ gene in $\mathrm{pF}: \mathrm{GFP} / \mathrm{CP}$ or the viral $\mathrm{CP}$ gene in pL:3a/GFP (Canto et al. 1997). Construct pF:3aGFP/CP contains sequences of an Fny-CMV RNA 3 in which the GFP gene has been fused to the $3^{\prime}$ end of the 3 a gene so that the two proteins are both expressed as a single fusion product (Canto et al. 1997). Construct pF:(M8)3a-GFP/CP was obtained from construct pFny309(M8) (Li 1995). This construct contains the sequence of Fny-CMV RNA 3, expressing a $3 \mathrm{a}$ protein mutated at sequences encoding amino acids 20 and 21 (both Asp to Ala). The construct was digested with Bam HI and NheI, and the resulting fragment containing the $5^{\prime}$ end of the $3 a$ gene, including mutation M8, was cloned into construct $\mathrm{pF}: 3 \mathrm{a}-\mathrm{GFP} / \mathrm{CP}$, linearized with the same enzymes. Construct pFL:(M8)3a/GFP contains sequences of a chimeric RNA 3 in which the first 642 nucleotides (containing the mutation M8 in the $\mathrm{N}$ terminus of the 3 a gene) were derived from Fny-CMV RNA 3 while the remaining sequences came from LS-CMV RNA 3. In addition, the GFP gene replaced the $\mathrm{CP}$ gene. This construct was obtained as follows: a DNA fragment, corresponding to the first 642 nucleotides of the viral RNA, was obtained after digestion with BamHI and NheI of the construct pFny309(M8). This DNA fragment was ligated into plasmid $\mathrm{pL}: 3 \mathrm{a} / \mathrm{GFP}$, previously digested with the same enzymes to generate construct pFL:N3(M8)/GFP. To confirm that no additional mutations had been introduced during the process of tagging the M8 3 a protein with GFP, the BamHI/NheI fragment containing the M8 mutation was isolated from this construct and substituted for the $3 \mathrm{a}$ gene in the full-length clone of Fny-CMV RNA 3 (pFny309), where the resulting virus obtained after inoculation of transcript RNAs showed the phenotype of M8 CMV. Construct $\mathrm{pF}: 3 \mathrm{a}-$ GFP/ $\triangle \mathrm{CP}$ was obtained from construct $\mathrm{pF}: 3 \mathrm{a}-\mathrm{GFP} / \mathrm{CP}$ by digestion with $S a l \mathrm{I}$ and $\mathrm{XhoI}$, and religation of the remaining plasmid. This removed most of the $\mathrm{CP}$ gene as an out-offrame deletion. Construct pFny309 $\Delta K p n I$ corresponded to Fny-CMV RNA 3 with a deletion of 501 nucleotides of the $3 \mathrm{a}$ gene (Kaplan et al. 1995).

The plasmids were linearized with PstI and the RNA transcripts were synthesized with T7 RNA polymerase as described previously (Zhang et al. 1994). Transcript mixtures were concentrated by ethanol precipitation prior to their use in mechanical inoculations. Plants were inoculated by gently rubbing the inocula (purified virus, viral RNA, or transcript RNAs) on aluminum oxide-dusted leaves.

\section{Protoplast isolation and electroporation.}

For the isolation of mesophyll protoplasts, plants were kept in a growth chamber for 5 days, at a constant temperature of $25^{\circ} \mathrm{C}$ and $16 \mathrm{~h}$ of daylight. Protoplasts were obtained from both tobacco and $N$. benthamiana plants, and $\left(10^{6}\right.$ protoplasts $)$ were electroporated with CMV transcript RNAs as described by Gal-On et al. (1994). Mannitol solutions of 10 and $12 \%$ were used for the isolation of protoplasts from tobacco and $N$. benthamiana, respectively. Protoplast cultures were kept undisturbed in a growth chamber at $25^{\circ} \mathrm{C}$, with $16 \mathrm{~h}$ of daylight, until their use in microscopic or protein analysis.

\section{Microscopy: detection of GFP fluorescence.}

Inoculated leaves and plant protoplasts were viewed under an epifluorescence microscope (Nikon, Tokyo). For GFP detection, an FITC/TRITC (rhodamine) multiband filter set was used (catalog no. 51004; Chroma Technologies, Brattleboro, VT). Fluorescence was also detected and recorded with an MRC 1000 confocal laser scanning microscope (Bio-Rad, Hercules, CA) with excitation and emission at $522 \mathrm{~nm}$. In 
each protoplast sample, at least 100 green fluorescent protoplasts were screened for the presence of fluorescent tubules. Each experiment was done at least three times. In inoculated leaves, where screening for the presence of fluorescent foci (composed of either single epidermal cells or clusters of mesophyll plus epidermal cells) was done, in each experiment, either the full leaf was screened, or up to 100 fluorescent foci. Digital images obtained with the confocal microscope were processed with Adobe Photoshop software (Adobe Systems, San Jose, CA).

\section{Protein analysis.}

For protein analysis, samples were extracted from $10^{6}$ protoplasts or plant leaves by grinding them in Laemmli buffer, subjected to sodium dodecyl sulfate-polyacrylamide gel electrophoresis (SDS-PAGE) and electroblotting to nitrocellulose membranes as described by Sambrook et al. (1989). The membranes were immunoprobed with antibodies against the 3a protein and against the viral CP of CMV, as previously described (Gal-On et al. 1994; Kaplan et al. 1995).

\section{ACKNOWLEDGMENTS}

We thank Qiubo Li for kindly providing the plasmids corresponding to full-length Fny-CMV RNA 3 with 3a protein mutations (M1 to M9). We also thank B. Ding for kindly providing us the transgenic tobacco expressing the 3a-GFP fusion protein. This work was supported by a grant-in-aid from the Scottish Office Agriculture, Environment and Fisheries Department.

\section{LITERATURE CITED}

Blackman, L. M., Boevink, P., Santa Cruz, S., Palukaitis, P., and Oparka, K. J. 1998. The movement protein of cucumber mosaic virus traffics into sieve elements in minor veins of Nicotiana clevelandii. Plant Cell 10:525-537.

Boccard, F., and Baulcombe, D. 1993. Mutational analysis of cis-acting sequences and gene function in RNA 3 of cucumber mosaic virus. Virology 193:563-578.

Canto, T., Prior, D. A. M., Hellwald, K.-H., Oparka, K. J., and Palukaitis, P. 1997. Characterization of cucumber mosaic virus IV. Movement protein and coat protein are both essential for cell-to-cell movement of cucumber mosaic virus. Virology 237:237-248.

Carrington, J. C., Kasschau, K. D., Mahajan, S. K., and Schaad, M. C. 1996. Cell-to-cell and long-distance transport of viruses in plants. Plant Cell 8:1669-1681.

Citovsky, V., Knorr, D., Schuster, G., and Zambryski, P. 1990. The P30 movement protein of tobacco mosaic virus is a single-stranded nucleic acid binding protein. Cell 4:637-647.

Citovsky, V., and Zambryski, P. 1991. How do plant virus nucleic acids move through intercellular connections? BioEssays 13:373-379.

Ding, B., Li, Q., Nguyen, L., Palukaitis, P., and Lucas, W. J. 1995. Cucumber mosaic virus 3 a protein potentiates cell-to-cell trafficking of CMV RNA in tobacco plants. Virology 207:345-353.

Gal-On, A., Kaplan, I., Roossinck, M. J., and Palukaitis, P. 1994. The kinetics of infection of zucchini squash by cucumber mosaic virus indicate a function for RNA 1 in virus movement. Virology 205:280289.

Grieco, F., Castellano, M. A., Di Sebastiano, G. P., Maggipinto, G., Neuhaus, J.-M., and Martelli, G. P. 1999. Subcellular localization and in vivo identification of the putative movement protein of olive latent virus 2. J. Gen. Virol. 80:1103-1109.

Heinlein, M., Padgett, H. S., Gens, J. S., Pickard, B. G., Casper, S. J., Epel, B. L., and Beachy, R. N. 1998. Changing patterns of localization of the tobacco mosaic virus movement protein and replicase to the endoplasmic reticulum and microtubules during infection. Plant Cell 10:1107-1120.

Itaya, A., Hickman, H., Bao, Y., Nelson, R., and Ding, B. 1997. Cell-to- cell trafficking of cucumber mosaic virus movement protein: Green fluorescent protein fusion produced by biolistic bombardment in tobacco. Plant J. 12:1223-1230.

Jansen, K. A. J., Wolfs, C. J. A. M., Lohuis, H., Goldbach, R. W., and Verduin, B. J. M. 1998. Characterization of the brome mosaic virus movement protein expressed in E. coli. Virology 242:387-394.

Kaplan, I. B., Shintaku, M. H., Li, Q., Zhang, L., Marsh, L. E., and Palukaitis, P. 1995. Complementation of virus movement in transgenic tobacco expressing the cucumber mosaic virus 3 a protein. Virology 209:188-199.

Kaplan, I. B., Zhang, L., and Palukaitis, P. 1998. Characterization of cucumber mosaic virus V. Cell movement requires capsid protein but not virions. Virology 246:221-231.

Kasteel, D. T. J., van der Wel, N. N., Jansen, K. A. J., Goldbach, R. W., and van Lent, J. W. M. 1997a. Tubule-forming capacity of the movement proteins of alfalfa mosaic virus and brome mosaic virus. J. Gen. Virol. 78:2089-2093.

Kasteel, D. T. J., Wellink, J., Goldbach, R. W., and van Lent, J. W. M. 1997b. Isolation and characterization of tubular structures of cowpea mosaic virus. J. Gen. Virol. 78:3167-3170.

Kikkert, M., van Poelwijk, F., Storms, M., Kassies, W., Bloksma, H., van Lent, J., Kormelink, R., and Goldbach, R. 1997. A protoplast system for studying tomato spotted wilt virus infection. J. Gen. Virol. 78:1755-1763.

Kormelink, R., Storms, M., van Lent, J., Peters, D., and Goldbach, R. 1994. Expression and subcellular localization of the $\mathrm{NS}_{\mathrm{M}}$ protein of tomato spotted wilt virus (TSWV), a putative viral movement protein. Virology 200:56-65.

Kragler, F., Monzer, J., Shash, K., Xoconostle-Cázares, B., and Lucas, W. J. 1998. Cell-to-cell transport of proteins: Requirement for unfolding and characterization of binding to a putative plasmodesmal receptor. Plant J. 15:367-381.

Li, Q. 1995. In vivo and in vitro characterization of the movement protein of cucumber mosaic virus. Ph.D. thesis. Cornell University, Ithaca, NY.

Li, Q., and Palukaitis, P. 1996. Comparison of the nucleic acid- and NTP-binding properties of the movement protein of cucumber mosaic cucumovirus and tobacco mosaic tobamovirus. Virology 216:71-79.

Linstead, P. J., Hills, G. J., Plaskitt, K. A., Wilson, I. G., Harker, C. L., and Maule, A. J. 1988. The subcellular localisation of the gene I product of cauliflower mosaic virus is consistent with a fraction associated with virus spread. J. Gen. Virol. 89:1809-1818.

Oparka, K. J., Boevink, P., and Santa Cruz, S. 1996. Studying the movement of plant viruses using the green fluorescent protein. Trends Plant Sci. 1:412-418.

Perbal, M. C., Thomas, C. L., and Maule, A. J. 1993. Cauliflower mosaic virus gene I product (P1) forms tubular structures which extend from the surface of infected protoplasts. Virology 195:281-285.

Rao, A. L. N. 1997. Molecular studies on bromovirus capsid protein III. Analysis of cell-to-cell movement competence of coat protein defective variants of cowpea chlorotic mottle virus. Virology 232:385-395.

Rizzo, T. M., and Palukaitis, P. 1990. Construction of full-length cDNA clones of cucumber mosaic virus RNAs 1, 2 and 3: Generation of infectious RNA transcripts. Mol. Gen. Genet. 222:249-256.

Roberts, A. G., Santa Cruz, S., Prior, D. A. M., Turgeon, R., and Oparka, K. J. 1997. Phloem unloading in sink leaves of Nicotiana benthamiana: Comparison of a fluorescent solute with a fluorescent virus. Plant Cell 9:1381-1396.

Ryabov, E. V., Roberts, I. M., Palukaitis, P., and Taliansky, M. 1999. Host-specific cell-to-cell and long-distance movements of cucumber mosaic virus are facilitated by the movement protein of groundnut rosette virus. Virology 260:98-108.

Sambrook, J., Fritsch, E. F., and Maniatis, T. A. 1989. Molecular Cloning: A Laboratory Manual. 2nd ed. Cold Spring Harbor Laboratory, Cold Spring Harbor, NY.

Schmitz, I., and Rao, A. L. N. 1996. Molecular studies on bromovirus capsid protein. I. Characterization of cell-to-cell movement-defective RNA 3 variants of brome mosaic virus. Virology 226:281-293.

Schoumacher, F., Erny, C., Berna, A., Godefroy-Colburn, T., and StussiGaraud, C. 1992a. Nucleic acid binding properties of the alfalfa mosaic virus movement protein produced in yeast. Virology 188:896899.

Schoumacher, F., Gagey, M.-J., Maira, M., Stussi-Garaud, C., and Godefroy-Colburn, T. 1992b. Binding of RNA by the alfalfa mosaic virus 
movement protein is biphasic. FEBS Lett. 308:231-234.

Storms, M. M. H., Kormelink, R., Peters, D., van Lent, J. W. M., and Goldbach, R. W. 1995. The nonstructural NSm protein of tomato spotted wilt virus induces tubular structures in plant and insect cells Virology 214:485-493.

Suzuki, M., Kuwata, S., Kataoka, J., Masuta, C., Nitta, N., and Takanami, Y. 1991. Functional analysis of deletion mutants of cucumber mosaic virus RNA 3 using an in vitro transcription system. Virology 183:106-113.

Thomas, C. L., and Maule, A. J. 1995. Identification of structural domains within the cauliflower mosaic virus movement protein by scanning deletion mutagenesis and epitope mapping. Plant Cell 7:561572.

van der Vossen, E. A. G., Neeleman, L., and Bol, J. F. 1994. Early and late functions of alfalfa mosaic virus coat protein can be mutated separately. Virology 202:891-903.

van Lent, J., Storms, M., van der Meer, F., Wellink, J., and Goldbach, R. 1991. Tubular structures involved in movement of cowpea mosaic virus are also formed in cowpea protoplasts. J. Gen. Virol. 72:26152623.

van Lent, J., Wellink, K. J., and Goldbach, R. 1990. Evidence for the involvement of the $58 \mathrm{~K}$ and $48 \mathrm{~K}$ proteins in the intercellular move- ment of cowpea mosaic virus. J. Gen. Virol. 71:219-223.

Vaquero, C., Sanz, A. I., Serra, M. T., and Garcia-Luque, I. 1996. Accumulation kinetics of CMV RNA 3-encoded proteins and subcellular localization of the 3 a protein in infected and transgenic plants. Arch. Virol. 141:987-999.

Vaquero, C., Turner, A. P., Demangeat, G., Sanz, A., Serra, M. T., Roberts, K., and Garcia-Luque, I. 1994. The 3a movement protein from cucumber mosaic virus increases the gating capacity of plasmodesmata in transgenic tobacco plants. J. Gen. Virol. 75:3193-3197.

Weiczorek, A., and Sanfaçon, H. 1993. Characterization and subcellular localization of tomato ringspot nepovirus putative movement protein. Virology 194:734-742.

Wellink, J., van Lent, J. W. M., Verver, J., Sijen, T., Goldbach, R. W., and van Kammen, A. 1993. The cowpea mosaic virus M RNAencoded 48 kilodalton protein is responsible for induction of tubular structures in protoplasts. J. Virol. 67:3660-3664.

Zhang, L., Hanada, K., and Palukaitis, P. 1994. Mapping local and systemic symptom determinants of cucumber mosaic cucumovirus in tobacco. J. Gen. Virol. 75:3185-3191.

Zheng, H., Wang, G., and Zhang, L. 1997. Alfalfa mosaic virus movement protein induces tubules in plant protoplasts. Mol. Plant-Microbe Interact. 10:1010-1014 